\title{
Genotyping and strain distribution of Mycobacterium avium subspecies hominissuis isolated from humans and pigs in Belgium, 2011-2013
}

C Vluggen $^{1}$, K Soetaert ${ }^{1}$, L Duytschaever ${ }^{23}$, J Denoël ${ }^{2}$, M Fauville-Dufaux ${ }^{1}$, F Smeets ${ }^{2}$, N Bruffaerts ${ }^{4}$, K Huygen ${ }^{4}$, D Fretin ${ }^{3}$,

L Rigouts ${ }^{56}$, C Saegerman ${ }^{2}$, V Mathys ${ }^{1}$

1. Bacterial Diseases Service, Operational Direction Communicable and Infectious Diseases, Scientific Institute of Public Health (WIV-ISP), Brussels, Belgium

2. Research Unit for Epidemiology and Risk Analysis applied to Veterinary Sciences (UREAR-ULg), Fundamental and Applied Research for Animal and Health (FARAH), Liège, Belgium

3. Bacterial Zoonoses of Livestock Unit, Operational Direction Bacterial Diseases, Veterinary and Agrochemical Research Centre (CODA-CERVA), Brussels, Belgium

4. Immunology Service, Operational Direction Communicable and infectious Diseases, Scientific Institute of Public Health (WIVISP), Brussels, Belgium

5. Mycobacteriology Unit, Institute of Tropical Medicine, Antwerp, Belgium

6. Department Biomedical Sciences, University of Antwerp, Belgium

Correspondence: Vanessa Mathys (vmathys@wiv-isp.be)

Citation style for this article:

Vluggen C, Soetaert K, Duytschaever L, Denoël J, Fauville-Dufaux M, Smeets F, Bruffaerts N, Huygen K, Fretin D, Rigouts L, Saegerman C, Mathys V. Genotyping and strain distribution of Mycobacterium avium subspecies hominissuis isolated from humans and pigs in Belgium, 2011-2013. Euro Surveill. $2016 ; 21$ (3):pii=30111. DOI: http://dx.doi.org/10.2807/1560-7917.ES.2016.21.3.30111

Article submitted on 09 December 2014 / accepted on 05 July 2015 / published on 21 January 2015

Mycobacterium avium represents a health concern for both humans and pigs. The characterisation of its subspecies is an important step improving the understanding of the epidemiology and the control of this pathogen. Ninety-two human $M$. avium strains were selected for a retrospective study. Subspecies determination by $r p o B$ sequencing and IS1245/IS901 analysis showed that $98.9 \%$ of Belgian human M. avium strains belong to the subspecies hominissuis (MAH). Some of these MAH strains present particular IS1245/IS901 profiles (absence of IS1245 and false IS901 detection provoked by the presence of ISMav6). In addition, $54 \mathrm{MAH}$ strains isolated from submandibular lymph nodes of Belgian pigs with lymphadenitis were included in this study. Genotyping of human and porcine isolates was performed using multispacer sequence typing (MST). In total, 49 different MST types were identified among pig $(n=11)$ and human $(n=43)$ MA isolates, with only five shared by both hosts. Among these MST types, 34 were newly identified. Our findings demonstrate the extensive genetic diversity among MAH isolates. Some genotypes were more prevalent in human or pigs but no correlation was observed between MST type and place of residence or the farm of origin for human and porcine isolates respectively, suggesting an environmental source of infection.

\section{Introduction}

Among non-tuberculous mycobacteria (NTM), Mycobacterium avium complex (MAC), composed of two major species $M$. avium and $M$. intracellulare, is the most frequently isolated from patients $[1,2]$ and the most common NTM identified as responsible of pulmonary disease [3]. In humans, lymphadenitis due to NTM primarily affects children and is caused by a variety of species, with predominance of M. avium [4]. In industrialised countries, in the absence of antiretroviral therapy, MAC is also recognised as the most common opportunistic bacterial infection in patients with advanced AIDS $[1,3]$, often with disseminated disease, rather than the typical lung involvement seen in immunocompetent patients [1].

The species M. avium, is divided into four subspecies; M. avium subsp. avium (MAA), M. avium subsp. silvaticum (MAS), M. avium subsp. paratuberculosis (MAP) and M. avium subsp. hominissuis (MAH) [5-7]. Recently, a phylogenetic study showed that MAH represents diverse groups of organisms from which two distinct groups, MAP and MAA/MAS, have evolved independently [8]. These subspecies of $M$. avium are genetically close, yet differ widely in their host range and pathogenicity. MAP is responsible for an intestinal illness in ruminants known as Johne's disease [9] and could be implicated in human Crohn's disease [10]. MAA and MAS mainly infect birds causing a tuberculosis-like disease, whereas $M A H$ is a frequent agent of human and pig mycobacterioses $[6,11]$. Although $\mathrm{MAH}$ can infect a wide variety of animals, pigs are its primary animal host species, causing granulomatous lesions mainly in the lymph nodes of the digestive tract, which can reduce the value of carcasses [12]. Moreover, an association between $\mathrm{MAH}$ and human lymphadenitis has been described [13]. MAH represents an increasing 


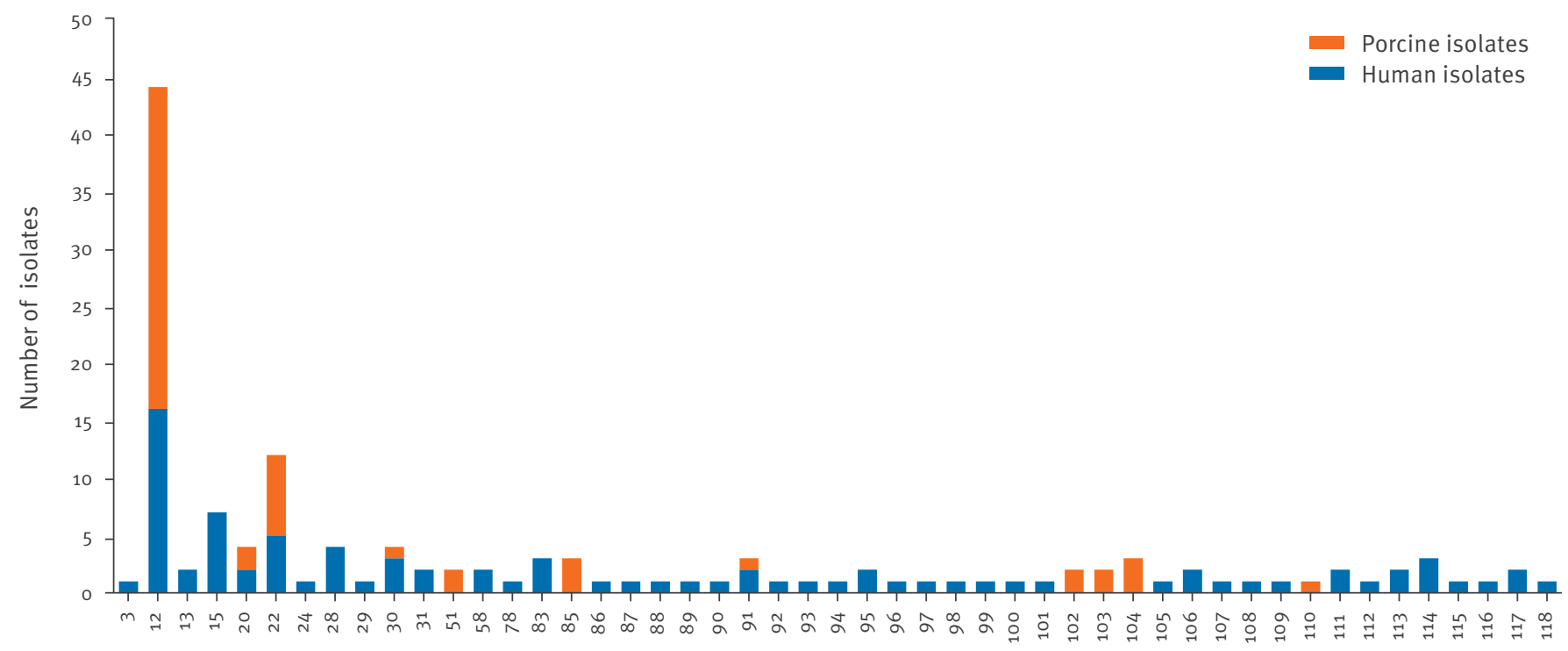

MST types

public health concern given its pathogenicity for both humans and pigs, and so precise characterisation of MA subspecies and genotypes is essential for epidemiological studies, identification of the source of infection, and to establish or exclude its possible zoonotic role $\left[14^{-16}\right]$.

In the period from 2010 to 2013, 2,964 NTM cultures were sent for identification to the Belgian National Reference Centre for Tuberculosis and Mycobacteria (WIV-ISP), of which 1,104 belonged to the M. avium complex, with the following distribution between the two species: 574 (19.4\% of the total) M. avium and 530 ( $17.9 \%$ of the total) $M$. intracellulare.

According to these data, M. avium is the predominant NTM isolated from Belgian patients. As the identification of $M$. avium isolates in Belgium is usually carried out by sequencing of the gene coding for 16SrRNA or by line probe assay (based on the analysis of the 23SrRNA gene), the distribution of the four M. avium subspecies remains largely unknown. In order to better characterise the $M$. avium strains circulating in Belgium, we selected clinical $M$. avium isolates from the above mentioned $M$. avium isolates and performed subspecies identification by sequencing of the $r p o B$ gene and analysis of the IS1245/IS901 elements. We also performed genotyping of the MAH isolates by using multispacer sequence typing (MST). As mycobacteriosis in pigs is mainly caused by MAH [16-18], we also genotyped previously identified $\mathrm{MAH}$ isolates from submandibular lymph nodes of Belgian pigs with lymphadenitis. The MST types of strains of human and porcine origin were compared.

\section{Materials and methods}

\section{Bacterial isolates and study design}

A total of 146 Belgian M. avium isolates from human patients $(n=92)$ and pigs $(n=54)$ were characterised in this study.

One hundred clinical $M$. avium isolates were selected from the collection maintained at WIV-ISP, Belgium. The isolates came from patients who had been diagnosed with $M$. avium in the last trimester of 2011 and in 2012, and for whom drug susceptibility testing (DST) had been requested after clinical, radiological and microbiological evaluation. Isolates were considered clinically relevant based on the criteria specified by the American Thoracic Society/Infectious Disease Society of America (ATS/IDSA) statement [19]. We aimed to exclude isolates representing environmental contamination of the samples. The selection represents $63 \%$ (100/159) of all M. avium isolates received at WIV-ISP for identification during the study period. WIV-ISP is the only Belgian laboratory performing DST for NTM. The M. avium species identification, carried out during routine activities at the reference laboratory, was performed by sequencing of the gene coding for the 16SrRNA, as previously described [20]. Eight isolates were eliminated from the study because of poor conservation quality, leaving a final sample of 92 human isolates.

The pig isolates were obtained from another ongoing study analysing submandibular lymph nodes from pigs with lymphadenitis sampled after veterinary inspection by the UREAR-ULg in all Belgian slaughterhouses. Isolates within the present study were sampled in 2012 and 2013. The first 54 M. avium isolates obtained were 
Mycobacterium avium subspecies identification of clinical isolates using IS1245/IS901 and rpoB sequencing, Belgium, 2011-2012, $(\mathrm{n}=92)$

\begin{tabular}{|c|c|c|c|c|}
\hline \multicolumn{3}{|c|}{$\begin{array}{l}\text { Interpretation based on } \\
\text { IS1245/IS901 detection }\end{array}$} & \multicolumn{2}{|r|}{$\begin{array}{l}\text { Interpretation based on } \\
\text { rpoB sequencing }\end{array}$} \\
\hline $\begin{array}{l}\text { No. of } \\
\text { isolates }\end{array}$ & IS1245/IS901 profile & Subspecies conclusion & $\begin{array}{l}\text { No. of } \\
\text { isolates }\end{array}$ & Perfect match with $r p o B$ sequence of \\
\hline 87 & IS1245 + / IS901- & $\begin{array}{l}\text { M. avium subsp. } \\
\text { hominissuis }\end{array}$ & $\begin{array}{c}72 \\
1 \\
1 \\
4 \\
9 \\
9\end{array}$ & $\begin{array}{c}\text { M. avium subsp. hominissuis } \\
\text { Mycobcterium sp. 09-7368 } \\
\text { Mycobacterium sp. 11-0068 (KPo98592) } \\
\text { Mycobacterium sp. 11-006596, 10-5763 or 09-4604 } \\
\text { rpoB PCR amplification impossible }\end{array}$ \\
\hline 3 & IS1245 + / IS901+ & $\begin{array}{l}\text { M. avium subsp. avium / } \\
\text { silvaticum }\end{array}$ & $\begin{array}{l}1 \\
2\end{array}$ & $\begin{array}{l}\text { Mycobacterium avium subsp. avium } \\
\text { Mycobacterium avium subsp. hominissuis }\end{array}$ \\
\hline 2 & IS1245 - / IS901+ & Not M. avium & 2 & M. avium subsp. hominissuis strain $\mathrm{TH}_{135}$ \\
\hline
\end{tabular}

IS: insertion sequence; M. avium: Mycobacterium avium; subsp.: subspecies; - : absence of the fragment; + : presence of the fragment.

included. They were isolated by the Belgian veterinary centre CODA-CERVA which supplied them for the study. $r p o B$ gene sequencing was used to confirm the subspecies identification that had been made by the CODACERVA using the IS1245/IS901 detection method. MST genotyping of these porcine isolates was performed as described above.

\section{Clinical data and patient information}

All clinical data recorded in the context of the present study had been collected not for research purposes but as part of the routine data collection for diagnosis. We collected the following data: origin of specimens (site of infection), date of collection, sex and age of patients and their city of residence (or postal code). Anonymity of data was ensured before analysis. The study was approved by the ethical committee of Hôpital Erasme (ULB) (reference P2014/028).

\section{IS901/IS1245 detection}

$M$. avium species identification of the selected strains was confirmed by PCR detection of the insertion sequence (IS) 1245 (present in M. avium strains), and the $M$. avium subspecies determined by analysing the presence/absence of IS901. Avian MAA and MAS strains contain IS901, whereas mammalian MAH and MAP strains lack it. The following primers were used for the analysis of IS1245 and IS901 respectively: IS1245F (5' AGGTGGCGTCGAGGAAGAC 3') and IS1245R (5' GCCGCCGAAACGATCTAC 3'), IS901F (5' GCAACGGTTGTTGCTTGAAAGGAAT 3') and IS901R (5' GCGCACGCATGATGAGTGGACTTAC 3'). Both PCR assays were performed as previously described [21,22]. PCR products were analysed by electrophoresis on a $2 \%$ agarose gel and visualised by staining with ethidium bromide and UV illumination. Sequencing of some IS901 amplicons was performed using the primers used for the PCR amplification.

\section{Gene sequencing}

Subspecies identification among $M$. avium isolates was also performed by sequencing of the $r p o B$ gene as described by Ben Salah et al. [23]. Briefly, the primers Myco-F (5' GGCAAGGTCACCCCGAAGGG 3') and Myco-R (5' AGCGGCTGCTGGGTGATCATC 3') were used to amplify a variable region of the $r p o B$ gene [23]. PCR products $(10 \mu \mathrm{l})$ were visualised on a $2 \%$ agarose gel after staining with ethidium bromide. The remaining PCR products were purified using a QIAquick 96 PCR Purification Kit (Qiagen) and sequenced with primers Myco-F and Mycoseq-R (5' GGACATCATCCTGAACACCCACG $\left.3^{\prime}\right)$. Sequencing was performed by using the BigDye Terminator Cycle Sequencing kit (Applied Biosystems) and analysed on an $A B I 3130 x /$ Sequencer (Applied Biosystems). The obtained forward and reverse sequences were re-assembled using the bioinformatics software Lasergene SeqMan Pro and used for sequence comparisons by BLAST with the NCBI database (National Centre for Biotechnology Information).

For a few strains, the subspecies identification was also confirmed by sequencing of the hsp65 gene using the classification proposed by Turenne et al. [24] and the method described by Dirac et al. [25].

\section{Multispacer sequence typing}

Genotyping of the M. avium isolates was performed by MST. The PCR amplification of the spacers MST2, MST4, MST15 and MST16 was performed as described by Cayrou et al. [26]. Amplicon purification and sequencing were performed as described above, with the same forward and reverse primers as used for the different MST PCRs.

The MST type was determined by consulting the MST database [27]. 
Statistical analysis

A $95 \%$ confidence interval $(\mathrm{Cl})$ of prevalence of clinical $M$. avium isolates according to the affected organ was estimated using an exact binomial distribution. The comparison of age between lymphadenitis and nonlymphadenitis patients was assessed using a two-sample t-test with unequal variances, whereas comparison of the sex ratio was assessed using the chi-squared test (Chi 2), and comparison of province of origin by the Fisher exact test [28]. The number of porcine $M$. avium strains according to the MST type and farm was assessed using a Poisson regression. A p value of 0.05 was considered as significant and all analyses were performed in Stata (StataCorp. 2011. Stata Statistical Software: Release 12. College Station, TX: StataCorp LP).

\section{Results}

\section{Clinical isolates}

The subspecies of $M$. avium isolates is not determined routinely in Belgium. In order to better characterise these $M$. avium isolates, a retrospective study was carried out $(n=92)$.

Information on the biological origin of the isolate was available for 71 patients. The vast majority of these were isolated from the respiratory tract $(n=51 ; 95 \% \mathrm{Cl}$ : 59.9-81.9) followed by lymph nodes $(n=13 ; 95 \% \mathrm{Cl}$ : 10.1-29.3), abscesses ( $n=4 ; 95 \% \mathrm{Cl}: 1.6-13.8)$, blood $(n=2 ; 95 \% C l: 0.3-9.8)$ and peritoneal fluid $(n=1 ; 95 \%$ $\mathrm{Cl}: 0.04-7.6)$.

The mean age of the selected patients was 58 years (data available for 90 of 92 patients). However, a significant difference in age was observed between lymphadenitis $(\mathrm{n}=13 ;$ mean $=15.3$ years, standard deviation $(S D)=22.8$ years) and non-lymphadenitis patients $(n=77$; mean $=65.1$ years, $S D=18.7$ years $)$ ( $p<0.0001$ ). The overall sex ratio was 1.04 (47 males and 45 females) but no significant difference was observed between patients with or without lymphadenitis ( $p$ value $=0.70$ ). In addition, lymphadenitis and non-lymphadenitis patients did not differ in geographic origin $(p$ value $=0.125)$.

\section{Subspecies identification}

Based on presence of IS1245 and absence of IS901 determined by PCR analysis, 87 isolates among the 92 analysed were identified as $\mathrm{MAH}$, and three as MAA/ MAS (Table 1). For the two remaining strains, conflicting results were observed between the IS1245 PCR (absence of the fragment usually present in $M$. avium strains) and sequencing of the 16sRNA used for the selection of the $M$. avium strains included in the study.

Subspecies identification based on the $r p o B$ sequence confirmed MAH subspecies for 72 of 87 isolates (Table 1). For the 15 remaining strains, nine were negative in $r p o B$ PCR amplification (repeated three times), five strains were identified as M. species (M. sp. 11-006596
(JF804804), M. sp. 10-5763 (JF327744) or M. sp. 09-4604 (JF327745) with same blast score, and M. sp. 09-7368 (JF437546)), and one had no perfect match with the $r p o B$ sequences currently recorded. The four $M$. species were isolated from a macaque, a pig, a cow and a steer, and characterised as close to $M$. avium subspecies in a study evaluating the usefulness of $r p o B$ sequencing for identification of Mycobacterium isolates of veterinary origin [29]. The new rpoB sequence discovered in this study was deposited in the GenBank database under accession number KPo98592. This strain was isolated from a lymph node of a 64 -year-old patient with lymphadenitis.

Of the three strains identified as MAA/MAS based on the presence of both IS1245 and IS901, one was confirmed by $r p o B$ analysis as MAA, and the other two were identified as MAH. We therefore sequenced the IS901 amplicons obtained for these three strains. Blast analysis of $173 \mathrm{nt}$ sequenced confirmed the presence of the IS901 in the isolate identified as MAA but revealed that one of the two MAH actually had the insertion sequence ISMav6, presenting 95\% similarity with IS901 [30].

Finally, for the two strains presenting negative results for the IS1245 amplification, the $r p o B$ sequence was identical to the MAH strain $\mathrm{TH}_{135}$ of the NCBI bank. hsp65 sequencing yielded codes 1 and 2 according to the classification of Turenne et al. [24]. These hsp65 codes have been described in MAH strains. It should be noted that these strains were also positive for the IS901 element, which is unusual for MAH strains. Sequencing of IS901 revealed the presence of insertion sequence ISMav6 instead of the standard IS901.

\section{Molecular typing}

The 92 human M. avium isolates included in this study were genotyped by MST in order to establish their genetic diversity (unrelated clinical isolates) or relatedness (clustered clinical isolates). MST types were interpretable for 87 strains. The distribution of the obtained genotypes is presented in the Figure. Overall, 43 different MST types were identified. Some genotypes were more prevalent such as MST type 12 found in 16 isolates, MST15 in seven isolates, MST22 in five isolates and MST28 in four isolates. Among the human isolates, twenty-nine MST types $(86,87,88,89,90,91,92,93$, 94, 95, 96, 97, 98, 99, 100, 101, 105, 106, 107, 108, 109, $111,112,113,114,115,116,117,118)$ were identified for the first time and recorded in the MST database developed by the Université de la Méditerranée. The MAA strain belonged to MST type 3, which was previously described as specific for MAA [26]. No association was observed between the MST types and sites of infection. Moreover, analysis of the geographical repartition of the patients revealed no correlation between the MST types and the patient's places/cities of residence. 
Repartition of the porcine Mycobacterium avium subspecies hominissuis isolates by multispacer sequencing type and farm, Belgium, 2012-2013

\begin{tabular}{|c|c|c|c|c|c|c|c|c|}
\hline \multirow{2}{*}{ Farm } & \multicolumn{8}{|c|}{ No. of porcine isolates } \\
\hline & MST 12 & MST 51 & MST20 & MST 103 & MST 22 & MST 104 & MST 102 & MST 51 \\
\hline 1 & 6 & & & 1 & & & & \\
\hline 2 & 2 & 1 & 1 & & & & & \\
\hline 3 & 1 & & & & & & & \\
\hline 4 & 2 & & & & & & & \\
\hline 5 & 1 & & & & & & & \\
\hline 6 & 6 & & & & & & & \\
\hline 7 & 10 & & & & & & & \\
\hline 8 & & 1 & & & & & & \\
\hline 9 & & 1 & & & & & & \\
\hline 10 & & & 1 & & & & & \\
\hline 11 & & & & 1 & & & & \\
\hline 12 & & & & & 1 & & & \\
\hline 13 & & & & & 6 & & & \\
\hline 14 & & & & & & 1 & & \\
\hline 15 & & & & & & 1 & 1 & \\
\hline 16 & & & & & & 1 & 1 & \\
\hline 17 & & & & & & & & 1 \\
\hline 18 & & & & & & & & 1 \\
\hline Total 18 & 28 & 3 & 2 & 2 & 7 & 3 & 2 & 2 \\
\hline
\end{tabular}

MST: multispacer sequencing type.

This table includes only MST types found in two or more isolates/pigs.

\section{Porcine isolates}

Within the ongoing Belgian project begun in 2012, the inspection of 125,000 randomly selected pig carcasses was performed by the UREAR-ULg. All submandibular lymph nodes from pigs with lymphadenitis were sampled and transmitted to CODA-CERVA. In the laboratory, a smear of all lymph nodes were stained by the Ziehl-Neelsen method and observed microscopically. The samples with acid-fast resistant stain, as well as a randomised selection of 266 samples selected to be able to detect an expected prevalence of gross lesions of $0.5 \%$ with an acceptable error of $0.06 \%$ and a confidence interval of $95 \%$, were cultivated on Coletsos (Biorad) and Löwenstein-Jensen (Biomérieux) media. The first 54 porcine $\mathrm{MAH}$ isolates were transmitted to the WIV-ISP, where 52 could be MST-typed, yielding 11 different MST types, of which five were also detected in human isolates. The most frequent porcine genotypes, MST type 12 (28 isolates) and MST type 22 (seven isolates), were also among the most frequently detected in humans. On the other hand, the second most prevalent human type (MST 15) was not detected in pigs. As for the clinical isolates, new MST types $(n=6)$ were identified $(85,91,102,103,104,110)$ and recorded in the MST database. The Figure shows the distribution of the MST types among porcine and human strains.
Analysis at fattening farm level (18 farms) was performed for all pigs infected with a MAH strain belonging to a MST type for which at least two strains were detected in this study. This analysis showed in all cases (six different MST types), that strains with the same MST type could be isolated from pigs originating from at least two different Belgian farms (Table 2). For example, the 28 porcine MST12 strains were isolated from pigs fattened at seven different farms. Farms of origin (farms of birth) supplying piglets to the fattening farms were also different in all cases. Moreover, MAH strains with different MST types were found among pigs originating from the same fattening farm. In addition, the number of different MAH strains isolated in a farm was not related to the MST type or the farm origin (Poisson regression; $p$ value > 0.12 ).

\section{Discussion}

MAC strains are the most frequently isolated NTM, and mycobacterial infections caused by these MAC strains are on the rise in both animals and humans. According to data from Belgium's National Reference Laboratory, M. avium currently represents about 20\% (167/857) of all NTM isolated from patients. In 2000 , this percentage was only $12 \%$ (63/506 NTM analysed) suggesting an increasing number of $M$. avium infections.

Although a true relative increase in $M$. avium infections is suspected, this observation could also be partially 
attributed to the increased awareness of NTM disease in recent years and the increased availability and sensitivity of laboratory techniques [3]. Indeed, the development of rapid liquid culture methods and molecular tests, such as species-specific PCR or reverse hybridisation assay, has improved and facilitated the detection and identification of NTM infections [3].

The present study was undertaken to characterise $M$. avium isolated from patients living in Belgium. First, the subspecies of a collection of clinical $M$. avium isolates was determined by detection of IS1245 and IS901, which is the most widely used technique for the (sub-)species identification of MAC. By using the strict IS1245/IS901 interpretation model, the majority $(87 / 92,94.6 \%)$ of human isolates were identified as MAH. When applying multiple identification strategies, however, conflicting data were obtained. Three of the five remaining isolates were detected as positive for IS901 and IS1245, which is the usual profile for MAA, but the MAA subspecies identification was only confirmed by $r p o B$ sequencing for one of these three isolates, whereas the other two yielded a typical MAH $r p o B$ profile. Moreover, the two other remaining isolates, showing positive results for IS901 but absence of amplicon for the IS1245 (usually used as a marker for M. avium strains) gave the identification MAH by hsp 65 sequencing and $\mathrm{MAH}$ strain $\mathrm{TH}_{135}$ by rpoB sequencing.

Strain $\mathrm{TH}_{135}$ was isolated in Japan from a HIV-negative patient with pulmonary disease. Genome analysis of this strain, carried out by Uchiya et al., revealed the presence of a new [31] insertion sequence, designated ISMav6, which presents $95 \%$ sequence identity with the subspecies marker IS901 [30]. PCR amplification of ISMav6 with primers designed for IS901 was previously reported, explaining the positive IS901 PCR results obtained for our two TH135 strains [32]. Moreover, M. avium isolates lacking IS1245 were also reported $[29,31,32]$. The existence of $M$. avium strains lacking IS1245 and MAH strains harbouring an IS homologous to IS901 complicates the interpretation of IS1245/901 results. In this study, we show that the presence of strains with this particular IS profile appears to be quite limited in Belgium with 2.2\% (2/92) of M. avium strains lacking IS1245 and 4.4\% (4/91) of MAH strains giving false IS901 PCR amplification. A more problematic situation was described in Japan with $16 \%$ and $67 \%$, respectively [32].

Taking into account the MAH identification by $r p o B$ sequencing for the four isolates presenting an unusual (but already described) IS1245/IS901 profile, 91 (98.9\%) of the 92 M. avium analysed isolates belong to the hominissuis subspecies, leaving only one isolate being identified as MAA. Contrary, subspecies identification based on $r p o B$ sequencing alone, would result in a lower percentage of MAH $(82.6 \% ; 76 / 92)$. The differences between the two techniques are caused by rрoB amplification problems [9] and isolates that are identified as $M$. species by $r p o B$ sequencing [6].
Regarding to the sampling design, different time periods in human (last trimester of 2011 and 2012) and pig (2012-2013) studies were applied for logistic reasons: availability of historical human samples and the opportunity of the ongoing pig study. These non-overlapping time frames may have caused a bias in inter-species strain diversity. To address this issue, human isolates from 2012 to 2013 should be analysed and compared with the current pig set.

Regardless of the technique used, we can conclude that the vast majority of $M$. avium strains isolated in Belgium belong to the subspecies hominissuis as described in other countries [13,32]. Our analysis also shows that application of either the IS1245/IS901 or $r p o B$ based strategy resulted in the determination of most $M$. avium subspecies, but it was the combination of the two techniques that allowed us to firmly conclude the species and/or subspecies identification for nineteen $(20.6 \%)$ cases.

Concerning the MAH genotypes, 48 different MST types were identified among pig and human $\mathrm{MAH}$ isolates, comprising five types shared by both hosts. Moreover, among these MST types, 34 were newly identified within this study (28 in human, five in porcine and one in both hosts) showing that the genotypes of the Belgian MAH strains are quite different from those isolated in other countries and previously recorded in the MST database. This clearly demonstrates the large genetic diversity of MAH isolates.

There was no correlation between the place of residence of the patients and the MST types of the MAH strains. Indeed, strains with same MST types were isolated from patients living in dispersed places in the country, and for patients living in the same city often various MST types were identified. Also for the porcine strains there was no correlation of MST type with the geographical localisation of the farms. Indeed, analysis of the MST types compared with the pig farms showed that MAH strains with identical MST types could be isolated from pigs originating from different fattening farms, while pigs on the same farms can be infected by MAH strains of various MST types. This genetic diversity of the MAH strains has also been described in other epidemiological studies (using other genotyping tools like IS1245-RFLP or MIRU-VNTR), suggesting an environmental source of infection rather than direct transmission between the hosts $[14,16,33]$. Alternatively, transmission from pigs to humans by consumption of meat could be considered. Indeed, in this situation, no correlation between pig farms and the patients' place of residence is needed. Although this route of transmission cannot be excluded, there is as yet no clear evidence of meat-borne transmission of M. avium to humans [34].

While considerable attempts were made to include only M. avium human isolates responsible for disease (and 
to avoid environmental contamination of the specimens) by selecting isolates of patients for whom DST had been requested by a physician, we consider it likely that the selection of pathogenic isolates studied may be underrepresented as some physicians probably treat their patients after species identification without asking the laboratory to perform DST. Another limitation of our study could be the small sample size, particularly due to the large genetic diversity detected among the MA strains. For instance, our data suggest that some MST types are only detected in M. avium isolated from humans or pigs. Analysis of a larger sample is required to formally test this hypothesis.

In conclusion, we report on the predominance of $\mathrm{MAH}$ among Belgian patients infected by a M. avium strain, and characterisation of Belgian MAH porcine isolates. Genotyping revealed a large genetic diversity of strains (whatever their origin; human or porcine) and the absence of a link between genotypes and the place of residence (human) or the farm of origin (pigs), suggesting an environmental source of infection. Some genotypes are however more frequent in human or in pigs, and some are only found in one of these two hosts. Moreover, the ISMav6 element was detected in some human isolates as described in another study [15]. As MAH represents an increasing public health concern, these host-strain particularities deserve more investigations.

\section{Acknowledgements}

This study was funded by the Federal Public Service, Health, Safety of the Food chain and Environment (convention RT 12/5 Lymphindic 1, Contractual Research, FPS Public Health, Security of the Food Chain and Environment). CV is a PhD student funded by the RP/PJ WIV-ISP (TYPTUB). The National Reference Centre for Tuberculosis \& Mycobacteria is partially supported by the Belgian Ministry of Social Affairs through a fund within the Health Insurance System. Gene sequencing was performed at the Platform Biotechnology and Molecular Biology at the Scientific Institute of Public Health (WIV-ISP)

\section{Conflict of interest}

None declared.

\section{Authors' contributions}

VM, KH, DF and CS conceived and designed the experiments; CV and KS performed the experiments; CV, VM and CS analysed the data; KS, LD, JD, FS, DF, CS contributed reagents/ materials/analysis tools; CV, LD, MFD, NB, KH, DF, LR, VM and CS wrote the paper.

\section{References}

1. Falkinham JO. Epidemiology of infection by nontuberculous mycobacteria.Clin Microbiol Rev. 1996;9(2):177-215.PMID: 8964035

2. Winthrop KL. Pulmonary disease due to nontuberculous mycobacteria: an epidemiologist's view. Future Microbiol. 2010;5(3):343-5. DOI: 10.2217/fmb.10.13 PMID: 20210543
3. Cook JL. Nontuberculous mycobacteria: opportunistic environmental pathogens for predisposed hosts. Br Med Bull. 2010;96(1):45-59. DOI: 10.1093/bmb/ldq035 PMID: 20977990

4. van Ingen J, Wisselink HJ, van Solt-Smits CB, Boeree MJ, van Soolingen D. Isolation of mycobacteria other than Mycobacterium avium from porcine lymph nodes.Vet Microbiol. 2010;144(1-2):250-3. DOI: 10.1016/j.vetmic.2009.12.047 PMID: 20097017

5. Thorel MF, Krichevsky M, Lévy-Frébault VV. Numerical taxonomy of mycobactin-dependent mycobacteria, emended description of Mycobacterium avium, and description of Mycobacterium avium subsp. avium subsp. nov., Mycobacterium avium subsp. paratuberculosis subsp. nov., and Mycobacterium avium subsp. silvaticum subsp. nov.Int Syst Bacteriol. 1990;40(3):254-60. DOI: 10.1099/00207713-403-254 PMID: 2397193

6. Mijs W, de Haas P, Rossau R, Van der Laan T, Rigouts L, Portaels F, et al. Molecular evidence to support a proposal to reserve the designation Mycobacterium avium subsp. avium for bird-type isolates and ' $M$. avium subsp. hominissuis' for the human/porcine type of M. avium. Int J Syst Evol Microbiol. 2002;52(Pt 5):1505-18.PMID: 12361252

7. Turenne CY, Wallace R, Behr MA. Mycobacterium avium in the postgenomic era.Clin Microbiol Rev. 2007;20(2):205-29. DOI: 10.1128/CMR.00036-06 PMID: 17428883

8. Turenne CY, Collins DM, Alexander DC, Behr MA. Mycobacterium avium subsp. paratuberculosis and M. avium subsp. avium are independently evolved pathogenic clones of a much broader group of M. avium organisms.J Bacteriol. 2008;190(7):2479-87. DOI: 10.1128/JB.01691-07 PMID: 18245284

9. Harris NB, Barletta RG. Mycobacterium avium subsp. paratuberculosis in Veterinary Medicine.Clin Microbiol Rev. 2001;14(3):489-512. DOI: 10.1128/CMR.14·3.489-512.2001 PMID: 11432810

10. Feller M, Huwiler K, Stephan R, Altpeter E, Shang A, Furrer $\mathrm{H}$, et al. Mycobacterium avium subspecies paratuberculosis and Crohn's disease: a systematic review and meta-analysis. Lancet Infect Dis. 2007;7(9):607-13. DOI: 10.1016/S14733099(07)70211-6 PMID: 17714674

11. Ignatov D, Kondratieva E, Azhikina T, Apt A. Mycobacterium avium-triggered diseases: pathogenomics.Cell Microbiol. 2012;14(6):808-18. DOI: 10.1111/j.1462-5822.2012.01776.x PMID: 22348543

12. Álvarez I, Castellanos E, Romero B, Aranaz A, Bezos I, Rodríguez S, et al. Epidemiological investigation of a Mycobacterium avium subsp. hominissuis outbreak in swine. Epidemiol Infect. 2011;139(1):143-8. DOI: 10.1017/ So950268810001779 PMID: 20653991

13. Despierres L, Cohen-Bacrie S, Richet H, Drancourt M. Diversity of Mycobacterium avium subsp. hominissuis mycobacteria causing lymphadenitis, France.Eur J Clin Microbiol Infect Dis. 2012;31(7):1373-9. DOI: 10.1007/s10096-011-1452-2 PMID: 22042560

14. Pate $M$, Kušar D, Zolnir-Dovč $M$, Ocepek M. MIRU-VNTR typing of Mycobacterium avium in animals and humans: heterogeneity of Mycobacterium avium subsp. hominissuis versus homogeneity of Mycobacterium avium subsp. avium strains. Res Vet Sci. 2011;91(3):376-81. DOI: 10.1016/j.rvSC.2010.10.001 PMID: 21047662

15. Iwamoto T, Nakajima C, Nishiuchi Y, Kato T, Yoshida S, Nakanishi N, et al. Genetic diversity of Mycobacterium avium subsp. hominissuis strains isolated from humans, pigs, and human living environment. Infect Genet Evol. 2012;12(4):846 52. DOI: 10.1016/j.meegid.2011.06.018 PMID: 21745597

16. Muwonge A, Oloya J, Kankya C, Nielsen S, Godfroid J, Skjerve $\mathrm{E}$, et al. Molecular characterization of Mycobacterium avium subspecies hominissuis isolated from humans, cattle and pigs in the Uganda cattle corridor using VNTR analysis. Infect Genet Evol. 2014;21:184-91. DOI: 10.1016/j.meegid.2013.11.012 PMID: 24270015

17. Cvetnić Z, Spicić S, Benic M, Katalinić-Janković V, Pate M, Krt B, et al. Mycobacterial infection of pigs in Croatia. Acta Vet Hung. 2007;55(1):1-9. DOI: 10.1556/AVet.55.2007.1.1 PMID: 17385551

18. Agdestein A, Johansen TB, Polaček V, Lium B, Holstad $\mathrm{G}$, Vidanović D, et al. Investigation of an outbreak of mycobacteriosis in pigs. BMC Vet Res. 2011;7(1):63. DOI: 10.1186/1746-6148-7-63 PMID: 22014189

19. ATS Mycobacterial Diseases Subcommittee,Griffith DE, Aksamit T, Brown-Elliott BA, Catanzaro A, Daley C, Gordin F, et al. . An official ATS/IDSA statement: diagnosis, treatment, and prevention of nontuberculous mycobacterial diseases. Am J Respir Crit Care Med. 2007;175(4):367-416. DOI: 10.1164/ rccm.200604-571ST PMID: 17277290

20. Kirschner P, Springer B, Vogel U, Meier A, Wrede A, Kiekenbeck $M$, et al. Genotypic identification of mycobacteria by nucleic 
acid sequence determination: report of a 2-year experience in a clinical laboratory. J Clin Microbiol. 1993;31(11):2882-9.PMID: 7505291

21. Guerrero C, Bernasconi C, Burki D, Bodmer T, Telenti A. A novel insertion element from Mycobacterium avium, IS1245 is a specific target for analysis of strain relatedness.J Clin Microbiol. 1995;33(2):304-7.PMID: 7714183

22. Godfroid J, Delcorps C, Irenge LM, Walravens K, Marché S, Gala JL. Definitive differentiation between single and mixed mycobacterial infections in red deer (Cervus elaphus) by a combination of duplex amplification of p34 and $\mathrm{f}_{57}$ sequences and Hpy 188 I enzymatic restriction of duplex amplicons.J Clin Microbiol. 2005;43(9):4640-8. DOI: 10.1128/JCM.43.9.46404648.2005 PMID: 16145120

23. Ben Salah I, Adékambi T, Raoult D, Drancourt M. rpoB sequence-based identification of Mycobacterium avium complex species. Microbiology. 2008;154(Pt 12):3715-23. DOI: 10.1099/mic.0.2008/020164-0 PMID: 19047739

24. Turenne CY, Semret M, Cousins DV, Collins DM, Behr MA. Sequencing of hsp 65 distinguishes among subsets of the Mycobacterium avium complex.J Clin Microbiol. 2006;44(2):433-40. DOI: 10.1128/JCM.44-2.433-440.2006 PMID: 16455896

25. Dirac MA, Weigel KM, Yakrus MA, Becker AL, Chen HL, Fridley G, et al. Shared Mycobacterium avium genotypes observed among unlinked clinical and environmental isolates. Appl Environ Microbiol. 2013;79(18):5601-7. DOI: 10.1128/ AEM.01443-13 PMID: 23851084

26. Cayrou C, Turenne C, Behr MA, Drancourt M. Genotyping of Mycobacterium avium complex organisms using multispacer sequence typing. Microbiology. 2010;156(Pt 3):687-94. DOI: 10.1099/mic.0.033522-o PMID: 19926652

27. Méditerranée infection. URMS Database. MST Mycobacterium avium. Marseille: Méditerranée infection. [Accessed 9 May 2014]. Available from: http://www mediterranee-infection com/ article php?laref $=254$ \&titre $=$ mst-mycobacterium -avium

28. Petrie A, Watson P. Additional techniques. In: Statistics for Veterinary and Animal Science. 2nd ed. Oxford: Blackwell Publishing; 2006. p. 191-211.

29. Higgins J, Camp P, Farrell D, Bravo D, Pate M, Robbe-Austerman $\mathrm{S}$. Identification of Mycobacterium spp. of veterinary importance using rpoB gene sequencing.BMC Vet Res. 2011;7(1):77. DOI: 10.1186/1746-6148-7-77 PMID: 22118247

30. Uchiya K, Takahashi H, Yagi T, Moriyama M, Inagaki $\mathrm{T}$, Ichikawa K, et al. Comparative genome analysis of Mycobacterium avium revealed genetic diversity in strains that cause pulmonary and disseminated disease. PLOS ONE. 2013;8(8):e71831. DOI: 10.1371/journal.pone.0071831 PMID: 23990995

31. Beggs ML, Stevanova R, Eisenach KD. Species identification of Mycobacterium avium complex isolates by a variety of molecular techniques.J Clin Microbiol. 2000;38(2):508-12. PMID: 10655336

32. Ichikawa K, Yagi T, Moriyama M, Inagaki T, Nakagawa T, Uchiya K, et al. Characterization of Mycobacterium avium clinical isolates in Japan using subspecies-specific insertion sequences, and identification of a new insertion sequence, ISMav6. J Med Microbiol. 2009;58(Pt 7):945-50. DOI: 10.1099/ jmm.0.008623-o PMID: 19502362

33. Bruijnesteijn van Coppenraet LE, de Haas PE, Lindeboom JA, Kuijper EJ, van Soolingen D. Lymphadenitis in children is caused by Mycobacterium avium hominissuis and not related to 'bird tuberculosis'. Eur J Clin Microbiol Infect Dis. 2008;27(4):293-9. DOI: 10.1007/S10096-007-0440-Z PMID: 18320245

34. European Food Safety Authority (EFSA),. Technical specifications on harmonised epidemiological indicators for public health hazards to be covered by meat inspection of swine.EFSA Journal.2011;9(10):2371.

\section{License and copyright}

This is an open-access article distributed under the terms of the Creative Commons Attribution (CC BY 4.0) Licence. You may share and adapt the material, but must give appropriate credit to the source, provide a link to the licence, and indicate if changes were made.

This article is copyright of the authors, 2016. 